\title{
Systemic Management of Colorectal Cancer
}

\author{
Presented by Wells A. Messersmith, MD
}

\begin{abstract}
Advances have been made in the systemic treatment of colorectal cancer, with approximately 12 chemotherapy or biologic agents approved for use as a single agent or in a combination. However, numerous gaps in our understanding of the disease remain, such as the lack of benefit with biologics in the adjuvant setting, the absence of biomarkers for most systemic therapies, and the reason why left-sided and right-sided tumors behave differently. At the 22nd NCCN Annual Conference, Dr. Wells A. Messersmith presented several impactful updates to the 2017 NCCN Clinical Practice Guidelines in Oncology for Colon Cancer and reviewed the outcomes with a host of therapies used for both early-stage and metastatic disease.
\end{abstract}

J Natl Compr Canc Netw 2017;15(5.5):699-702

\begin{abstract}
"Y ou really can't treat colorectal cancer [CRC] today without molecular testing," remarked Wells A. Messersmith, MD, Co-Director of the Developmental Therapeutics Program, University of Colorado Cancer Center, and Professor of Medicine, Director of the Gastrointestinal Cancer Program, and Division Head of Medical Oncology, University of Colorado School of Medicine. CRCs are associated with complex genetics, and the presence of KRAS/NRAS mutations (among others) plays a key role in selecting treatment with the best chance of success.
\end{abstract}

\section{Adjuvant Therapy}

Although 11 agents have been approved by the FDA for CRC, only 3 are used in the adjuvant setting (fluorouracil [5-FU], capecitabine, and oxaliplatin) and only 2 (cetuximab and panitumumab) are biomarkerdriven, said Dr. Messersmith, who also is a member of the NCCN Guidelines Panel for Colon/Rectal/Anal Cancers. In addition, the use of programmed cell death protein 1 (PD-1) inhibitors pembrolizumab and

\footnotetext{
Presented by Wells A. Messersmith, MD, Division of Medical Oncology, University of Colorado Cancer Center, Aurora, Colorado.

$\mathrm{Dr}$. Messersmith has disclosed that he receives grant/research support from Alexo Therapeutics, Inc.; Bayer HealthCare; Genentech, Inc.; Immunomedics, Inc.; Millennium Pharmaceuticals, Inc.; OncoMed Pharmaceuticals; Pfizer Inc.; and Roche Laboratories, Inc. He serves as a scientific advisor for Gilead (Data Safety Monitoring Board).

Correspondence: Wells A. Messersmith, MD, University of Colorado Cancer Center, Division of Medical Oncology, Mailstop 8117, 12801 East 17th Avenue, L18-8121, Aurora, CO 80045. E-mail: wells.messersmith@ ucdenver.edu
}

nivolumab are incorporated in the NCCN Guidelines, despite not being FDA-approved for this indication, because the data are "so compelling," he added.

Dr. Messersmith focused mainly on 2 combination therapies for adjuvant chemotherapy for resected (primary) colon cancer: FOLFOX (leucovorin, 5-FU, oxaliplatin) and CAPOX (capecitabine and oxaliplatin). In the MOSAIC trial of $>2,200$ patients, ${ }^{1}$ 5-year diseasefree survival was better with the addition of oxaliplatin to infusional 5-FU/leucovorin in those with stage III disease, and there was a "hint" of a benefit in those with stage II disease; however, the overall survival (OS) benefit was confined to those patients with stage III disease. The FOLFOX regimen was more toxic, and few patients can tolerate 6 months of full-dose oxaliplatin. "Obviously, there is a price to be paid when you add on another drug," he added.

Similar improvement in disease-free survival in patients with stage III colon cancer was reported with CAPOX in a multicenter randomized trial. ${ }^{2}$ However, Dr. Messersmith mentioned that this trial was not a head-to-head comparison with FOLFOX. For some unknown reason, adding irinotecan to 5-FU/leucovorin has not been shown to be efficacious, he said.

Based on these results, both FOLFOX and CAPOX are category 1 recommendations for adjuvant chemotherapy after resection of stage III colon cancer in the 2017 NCCN Guidelines. ${ }^{3}$ However, Dr. Messersmith reminded, the risks and benefits must be weighed and discussed with patients before treatment. NCCN added the following caveats for decisions regarding adjuvant 
Messersmith

therapy for patients with stage II disease: "Don't use any adjuvant therapy for stage II microsatellite instability high (MSI-H) colon cancer; there are insufficient data to recommend use of multigene assay panels to determine benefit from adjuvant chemotherapy; there is a similar lack of data for 'high-risk' stage II disease and benefit from adjuvant chemotherapy." ${ }^{3}$

Finally, adding the biologic bevacizumab or cetuximab did not appear to offer an advantage in the adjuvant setting. Although targeted therapies play an important role in CRC, Dr. Messersmith stated they are "generally not used in the postoperative setting."

\section{Treatment of Advanced Disease}

The treatment algorithms in the 2017 NCCN Guidelines clearly distinguish between resectable/ curative disease and unresectable disease. For patients with resectable tumors, "You can go straight to resection or consider neoadjuvant chemotherapy," Dr. Messersmith said. For those with resectable metachronous metastases, primary resection is preferred, with consideration of local therapy or neoadjuvant chemotherapy.

The EORTC 40983 trial compared surgery alone with FOLFOX before and after surgery in patients with resectable liver metastases from CRC. ${ }^{4}$ The investigators found no statistical difference in OS between the treatments. However, the hazard ratio for progression-free survival $(\approx 0.8)$ was similar to that seen in the MOSAIC trial.

As for initially unresectable disease with metachronous metastases, the 2017 NCCN Guidelines allow for conversion therapy. "If you are able to convert patients from unresectable to resectable, you could actually save their lives," Dr. Messersmith announced.

Aggressive multiagent regimens may be more effective in the conversion setting, as shown by the small phase II METHEP trial which evaluated intensified chemotherapy in those with potentially resectable and unresectable liver metastases from CRC. ${ }^{5}$ Among the regimens studied, FOLFIRINOX (infusional leucovorin, 5-FU, irinotecan, and oxaliplatin) offered the best conversion rate to resectability $(67 \%)$.

Dr. Messersmith mentioned that, at this time, there is no proven role for biologic agents in the treatment of patients undergoing metastasectomy, especially following resection.

\section{Targeted Agents for Advanced Disease}

Agents that target the vascular endothelial growth factor (VEGF) receptor-bevacizumab, ramucirum$\mathrm{ab}$, and ziv-aflibercept-have shown an incremental benefit in the second-line metastatic setting, according to Dr. Messersmith. Modest improvements were noted in progression-free survival and OS, with remarkably similar hazard ratios with all 3 agents, he indicated. However, toxicity was an issue, and adverse events led to treatment discontinuation in some patients. Currently, there are no proven biomarkers for VEGF inhibitors.

Studies have also focused on targeting epidermal growth factor receptor (EGFR) in metastatic CRC, and molecular markers determine the benefit versus harm of EGFR-targeting agents. In one trial of cetuximab, ${ }^{6}$ patients with wild-type KRAS achieved a benefit, whereas those with a KRAS mutation did not. Dr. Messersmith pointed out that KRAS was not prognostic in this study, because it had no influence on survival among patients treated with best supportive care alone.

The CRYSTAL trial, ${ }^{7}$ in the first-line setting for metastatic CRC, showed similar results. The addition of cetuximab to FOLFIRI improved survival in patients with wild-type KRAS disease, but not in those with KRAS-mutant disease. In addition, this study showed that BRAF mutation was a negative prognostic indicator. "Regardless of treatment, BRAF-mutant patients did worse," stated Dr. Messersmith, "so this seems to be an aggressive subtype of CRC in need of better treatments." Follow-up studies have shown that mutations in NRAS, as well as other codons of KRAS aside from 12/13 in the initial reports, also do not derive benefit from EGFRtargeting monoclonal antibodies.

\section{Tumor Location Matters}

The prognostic and predictive role of tumor location in metastatic CRC has emerged recently, with the findings of the CALGB/SWOG 80405 trial. ${ }^{8}$ Patients with left-sided tumors had a better median OS than those with right-sided tumors ( 33.3 vs 19.4 months) when treated with the addition of cetuximab after FOLFIRI or FOLFOX. In fact, cetuximab was of no apparent benefit in patients with right-sided tumors. "This was performed in a first-line setting," reminded 
Dr. Messersmith, "so it is unclear whether [this finding] applies to other settings."

As a result of these findings, initial systemic therapy for advanced or metastatic colon cancer in the 2017 NCCN Guidelines was updated to include cetuximab or panitumumab as an appropriate option (in combination with a chemotherapy backbone), for KRAS/NRAS wild-type disease and left-sided tumors only (Figure 1). " "For right-sided tumors, you should stick with bevacizumab and triplet chemotherapy," suggested Dr. Messersmith. This distinction, however, is not noted under the second-line algorithm, pending further studies. "From my standpoint, I would avoid panitumumab and cetuximab no matter what line for right-sided tumors, until more studies are available."

\section{Mismatch-Repair Status and Immunotherapy}

"In most cases, CRC has been left out of the immunotherapy party, and it is unclear why that is," admitted Dr. Messersmith. However, immune checkpoint blockade has been found to be of clinical benefit in an MSI-H subgroup: those with mismatch repair (MMR)-deficient CRC tumors.

In a phase II trial by Le et al, ${ }^{9}$ pembrolizumab was evaluated in 41 patients with progressive metastatic carcinoma. "Very high partial response and stable disease rates were reported in those with MMRdeficient CRC, but in those with MMR-proficient tumors, they did not really see any activity," Dr. Messersmith said. Moreover, the duration of response may prove to be the key value of immunotherapy in this setting, "with some patients responding out to 730 days," he added. "In my own clinic, we have a number of patients on clinical trials responding for years."

Consequently, in the advanced or metastatic setting, the NCCN Guidelines now include nivolumab or pembrolizumab as an appropriate initial therapy only for patients with MMR-deficient/MSI-H disease. For this small population of approximately $5 \%$, cytotoxic combinations are not appropriate, added Dr. Messersmith. This recommendation is included in both the second- and third-line settings as well.

Thus, resected tumors should be tested for MSI. "We hope you come from institutions that do reflexive testing for MSI," Dr. Messersmith concluded.

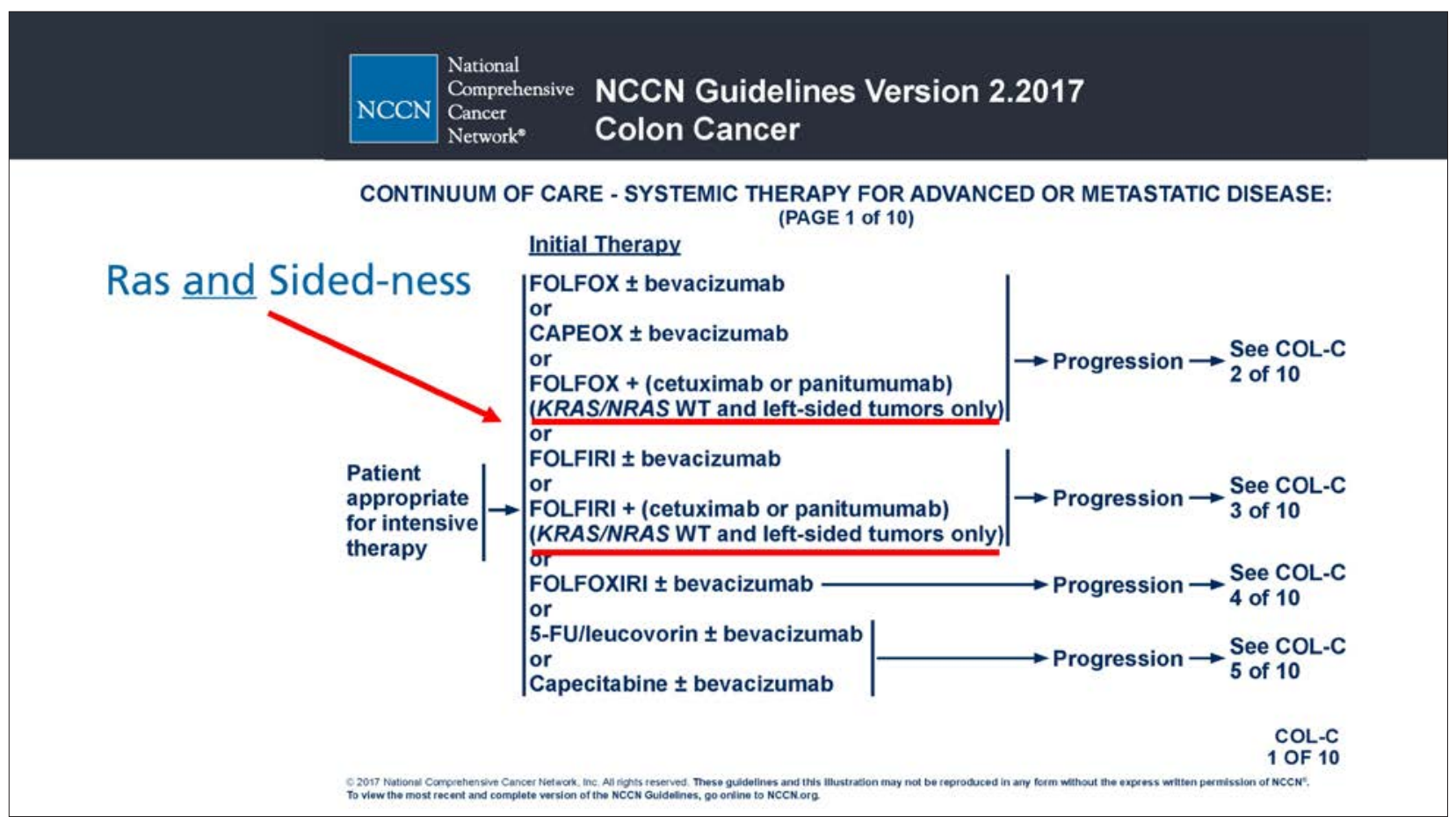

Figure 1. Continuum of care: systemic therapy for advanced or metastatic disease. 


\section{Messersmith}

\section{References}

1. André $T$, Boni $C$, Navarro $M$, et al. Improved overall survival with oxaliplatin, fluorouracil, and leucovorin as adjuvant treatment in stage II or III colon cancer in the MOSAIC trial. J Clin Oncol 2009;27:3109-3116.

2. Haller DG, Tabernero J, Maroun J, et al. Capecitabine plus oxaliplatin compared with fluorouracil and folinic acid as adjuvant therapy for stage III colon cancer. J Clin Oncol 2011;29:1465-1471.

3. Benson AB III, Venook AP, Cederquist L, et al. NCCN Clinical Practice Guidelines in Oncology: Colon Cancer, Version 2.2017. Accessed April 14, 2017. To view the most recent version of these guidelines, visit NCCN.org.

4. Nordlinger B, Sorbye $\mathrm{H}$, Glimelius B, et al. Perioperative FOLFOX4 chemotherapy and surgery versus surgery alone for resectable liver metastases from colorectal cancer (EORTC 40983): long-term results of a randomised, controlled, phase 3 trial. Lancet Oncol 2013;14:1208-1215.

5. Ychou M, Rivoire M, Thezenas S, et al. A randomized phase II trial of three intensified chemotherapy regimens in first-line treatment of colorectal cancer patients with initially unresectable or not optimally resectable liver metastases. The METHEP trial. Ann Surg Oncol 2013;20:4289-4297.

6. Karapetis CS, Khambata-Ford S, Jonker DJ, et al. K-ras mutations and benefit from cetuximab in advanced colorectal cancer. N Engl J Med 2008;359:1757-1765.

7. Van Cutsem E, Köhne CH, Láng I, et al. Cetuximab plus irinotecan, fluorouracil, and leucovorin as first-line treatment for metastatic colorectal cancer: updated analysis of overall survival according to tumor KRAS and BRAF mutation status. J Clin Oncol 2011;29:2011-2019.

8. Venook AP, Niedzwiecki D, Innocenti F, et al. Impact of primary $\left(1^{\circ}\right)$ tumor location on overall survival and progression-free survival in patients with metastatic colorectal cancer: analysis of CALGB/SWOG 80405 (Alliance) [abstract]. J Clin Oncol 2016;34(Suppl):Abstract 3504.

9. Le DT, Uram JN, Wang H, et al. PD-1 blockade in tumors with mismatchrepair deficiency. N Engl J Med 2015;372:2509-2520. 